Draft version August 13, 2018

Preprint typeset using $\mathrm{LATEX}_{\mathrm{E}} \mathrm{X}$ style emulateapj v. 2/16/10

\title{
RADIATION HYDRODYNAMICS SIMULATIONS OF PHOTOEVAPORATION OF PROTOPLANETARY DISKS II: METALLICITY DEPENDENCE OF UV AND X-RAY PHOTOEVAPORATION
}

\author{
Riouhei Nakatani ${ }^{1}$, Takashi Hosokawa ${ }^{2}$, Naoki Yoshida ${ }^{1,3,4}$, Hideko Nomura ${ }^{5}$, and Rolf Kuiper $^{6}$ \\ Draft version August 13, 2018
}

\begin{abstract}
We perform a suite of radiation hydrodynamics simulations of photoevaporating disks with varying the metallicity in a wide range of $10^{-3} Z_{\odot} \leq Z \leq 10^{0.5} Z_{\odot}$. We follow the disk evolution for over $\sim 5000$ years by solving hydrodynamics, radiative transfer, and non-equilibrium chemistry. Our chemistry model is updated from the first paper of this series by adding X-ray ionization and heating. We study the metallicity dependence of the disk photoevaporation rate and examine the importance of X-ray radiation. In the fiducial case with solar metallicity, including the X-ray effects does not significantly increase the photoevaporation rate when compared to the case with ultra-violet (UV) radiation only. At sub-solar metallicities in the range of $Z \gtrsim 10^{-1.5} Z_{\odot}$, the photoevaporation rate increases as metallicity decreases owing to the reduced opacity of the disk medium. The result is consistent with the observational trend that disk lifetimes are shorter in low metallicity environments. Contrastingly, the photoevaporation rate decreases at even lower metallicities of $Z \lesssim 10^{-1.5} Z_{\odot}$, because dust-gas collisional cooling remains efficient compared to far UV photoelectric heating whose efficiency depends on metallicity. The net cooling in the interior of the disk suppresses the photoevaporation. However, adding X-ray radiation significantly increases the photoevaporation rate, especially at $Z \sim 10^{-2} Z_{\odot}$. Although the $\mathrm{X}$-ray radiation itself does not drive strong photoevaporative flows, $\mathrm{X}$-rays penetrate deep into the neutral region in the disk, increase the ionization degree there, and reduce positive charges of grains. Consequently, the effect of photoelectric heating by far UV radiation is strengthened by the X-rays and enhances the disk photoevaporation.
\end{abstract}

Subject headings: protoplanetary disks - stars: formation - infrared: planetary systems - stars: pre-main-sequence - ultraviolet: stars

\section{INTRODUCTION}

Infrared observations of star-forming regions show that the fraction of infrared excess, a signature of the existence of protoplanetary disks (PPDs), decreases with the age of the system, suggesting a finite lifetime of $3-6$ Myr for the PPDs in solar metallicity environments (Haisch et al. 2001; Hernández et al. 2007; Meyer et al. 2007; Mamajek 2009; Fedele et al. 2010; Ribas et al. 2014). There are a few physical mechanisms proposed theoretically, which predict the disk dispersal time of a few million years, but the exact processes that drive destruction or evaporation of a PPD are poorly known. Interestingly, recent observations suggest that PPDs in sub-solar metallicity environments may have significantly shorter lifetimes of $\lesssim 1 \mathrm{Myr}$ (Yasui et al. 2009, 2010, 2016 a a).

Photoevaporation, a physical process with which

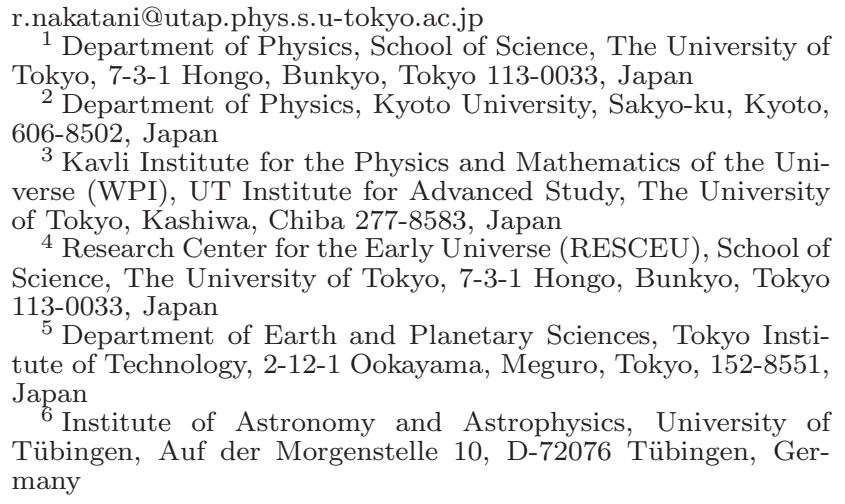

2 Department of Physics, Kyoto University, Sakyo-ku, Kyoto, 606-8502, Japan

${ }^{3}$ Kavli Institute for the Physics and Mathematics of the Universe (WPI), UT Institute for Advanced Study, The University of Tokyo, Kashiwa, Chiba 277-8583, Japan

${ }_{4}$ Research Center for the Early Universe (RESCEU), School of Science, The University of Tokyo, 7-3-1 Hongo, Bunkyo, Tokyo 113-0033, Japan

${ }^{5}$ Department of Earth and Planetary Sciences, Tokyo Institute of Technology, 2-12-1 Ookayama, Meguro, Tokyo, 152-8551, Japan

${ }^{6}$ Institute of Astronomy and Astrophysics, University of Tübingen, Auf der Morgenstelle 10, D-72076 Tübingen, Germany

outflows are excited by irradiation from the central star, is proposed as a promising disk dispersal mechanism (Hollenbach et al. 1994; Clarke et al. 2001; Alexander et al. 2004; Font et al. 2004; Ercolano et al. 2009; Gorti \& Hollenbach 2009; Owen et al. 2010). Farultraviolet (FUV; $6 \mathrm{eV} \lesssim h \nu \leq 13.6 \mathrm{eV}$ ), extremeultraviolet (EUV; $13.6 \mathrm{eV} \leq h \nu \lesssim 0.1 \mathrm{keV}$ ), and $\mathrm{X}$ rays $(0.1 \mathrm{keV} \leq h \nu \leq 10 \mathrm{keV})$ can cause photoevaporation through different physical processes. FUV radiation heats the disk gas by photoelectric heating and/or photopumping of $\mathrm{H}_{2}$ (Wang \& Goodman 2017), whereas photoionization heating by EUV and X-ray radiation can also drive evaporative flows from a PPD.

EUV radiation is mainly absorbed by hydrogen atoms in the disk gas, and the absorption cross section per hydrogen atom, of the order of $\sim 10^{-17} \mathrm{~cm}^{2}$, is much larger than those for FUV and X-ray radiation that are absorbed by dust grains and hydrogen/helium/heavy elements, respectively. Therefore, FUV and X-ray radiation penetrate the deep interior of a disk with column densities of $N_{\mathrm{H}} \sim 10^{21} \mathrm{~cm}^{-2}$, whereas EUV radiation effectively heats lower density regions close to the disk surfaces with column densities of $N_{\mathrm{H}} \sim 10^{19}-10^{20} \mathrm{~cm}^{-2}$.

Analytic models and numerical simulations suggest that EUV-driven photoevaporative flows originating from low-density regions (disk surfaces) yield a mass-loss rate of $\sim 10^{-10}-10^{-9} M_{\odot} \mathrm{yr}^{-1}$ (Hollenbach et al. 1994; Tanaka et al. 2013). It is smaller by a factor of ten to even hundreds than FUV- and X-ray-driven photoevaporation rates (Gorti \& Hollenbach 2009; Ercolano et al. 2009; Owen et al. 2010; Nakatani et al. 2018, hereafter 
Paper I). Recent studies on photoevaporation of a PPD by FUV and X-ray radiation show rather diverse results, and the relative importance of FUV and X-rays is under debate Gorti \& Hollenbach 2009; Ercolano et al. 2009: Owen et al.|2010, 2012; Gorti et al. 2015). Interestingly, Ercolano \& Clarke (2010) show that X-ray photoevaporation is more efficient with lower metallicities owing to the reduced opacity effect. Unfortunately, these previous studies adopt different stellar models, disk models, and even different sets of chemistry, and thus one cannot compare the results directly. Furthermore, simplified assumptions are often made such as hydrostatic disk structure and/or radiative equilibrium, which degrades the reality of the calculations when considering the actual, dynamic evolution of a PPD. In order to examine the effect of FUV and X-ray radiation on PPD photoevaporation, it is necessary to perform hydrodynamics simulations with all of the above physical processes included self-consistently.

Two recent studies, Wang \& Goodman (2017) and our Paper I, use hydrodynamics simulations with radiative transfer and non-equilibrium chemistry to follow the disk photoevaporation around a solar-type star. Both studies conclude that FUV photons effectively drive photoevaporation, although there are a few differences regarding the most effective heating process. In Paper I, we investigate metallicity dependence of UV photoevaporation rates. We conclude that the FUV-driven photoevaporation rate increases with decreasing metallicity for $10^{-0.5} Z_{\odot} \lesssim Z \lesssim 10 Z_{\odot}$. We also find that photoelectric heating due to FUV becomes inefficient as metallicity decreases, compared with dust-gas collisional cooling. This reduced FUV heating lowers the temperatures of the neutral region. For $Z \lesssim 10^{-1.5} Z_{\odot}$, the neutral region temperatures are too low for photoevaporative flows to be excited. Only EUV-driven photoevaporative flows contribute to the mass loss in this case, and thus the photoevaporation rates are smaller by about an order of magnitude than those with $Z \gtrsim 10^{-1} Z_{\odot}$. It is worth mentioning that the simulations of Wang \& Goodman (2017), which incorporate X-ray heating, show that the $\mathrm{X}$-ray radiation itself is not the primary cause of photoevaporation.

In the present study, we perform a suite of simulations of photoevaporating protoplanetary disks with various metallicities $10^{-3} Z_{\odot} \leq Z \leq 10^{0.5} Z_{\odot}$. Our simulations incorporate X-ray heating and ionization coupled with our chemistry model of Paper I. We examine how Xray radiation affects the disk photoevaporation rate, and determine the relative importance of FUV and X-ray in the process of photoevaporation. Also, we investigate the metallicity dependence of photoevaporation rates due to both UV and X-ray.

The paper is organized as follows. In Section 2, we present the methods of our simulations. In Section 3 , we discuss the simulation results. A final discussion and a summary are given in Section 4 and Section 5. respectively.

\section{METHODS}

We perform a suite of simulations of photoevaporating protoplanetary disks with various metallicities of $10^{-3} Z_{\odot} \leq Z \leq 10^{0.5} Z_{\odot}$. We solve coupled equations of hydrodynamics, radiative transfer, and non-equilibrium chemistry.

We largely follow the method of Paper I, except that we include $\mathrm{X}$-ray radiation and add $\mathrm{H}_{2}{ }^{+}$as a chemical species in the present study. In this section, we briefly summarize our model and refer the readers to Paper I for numerical methods. Details of the X-ray implementation are described in Appendix A

We take into account FUV, EUV, and X-ray irradiation from the central star. The central star is assumed to have the stellar parameters tabulated in TABLE 1. Although the stellar properties may well depend on metallicity, we adopt the fixed parameters in all our simulations in order to make it easy to compare the results directly.

The FUV and EUV luminosities and the SED are the same as in Paper I. We adopt the X-ray SED presented in Nomura et al. (2007), which is derived by fitting the observational XMM-Newton data for TW Hydrae with using a two-temperature thin thermal plasma model (Mewe et al. 1985; Liedahl et al. 1995). In our model, we set the minimum and maximum energy of the SED to be $E_{\min }=0.1 \mathrm{keV}$ and $E_{\max }=10 \mathrm{keV}$, respectively 7 .

The disk gas is composed of the eight chemical species: $\mathrm{H}, \mathrm{H}^{+}, \mathrm{H}_{2}, \mathrm{H}_{2}^{+}, \mathrm{CO}, \mathrm{O}, \mathrm{C}^{+}$, and electrons. Note that we add $\mathrm{H}_{2}{ }^{+}$in order to follow $\mathrm{H}_{2}$ ionization by X-rays. Hereafter, we refer to $\mathrm{H}, \mathrm{H}^{+}, \mathrm{H}_{2}$, and $\mathrm{H}_{2}{ }^{+}$ as H-bearing species and $\mathrm{CO}, \mathrm{O}$, and $\mathrm{C}^{+}$as metal species. The amounts of the dust and heavy elements are determined by the ISM values for our solar metallicity disk, and assumed to be proportional to the ratio of the metallicity $Z$ to the local interstellar metallicity $Z_{\odot}$. Thus, we use the dust to gas mass ratio $\mathscr{D} \mathscr{G}=0.01 \times Z / Z_{\odot}$, and the gas-phase elemental abundances of carbon $y_{\mathrm{C}}=0.927 \times 10^{-4} Z / Z_{\odot}$ and oxygen $y_{\mathrm{O}}=3.568 \times 10^{-4} Z / Z_{\odot}$ (Pollack et al. 1994; Omukai 2000). The dust-to-gas mass ratio and the elemental abundances are the same as in Paper I.

TABLE 1

Properties of THE MOdeL

\begin{tabular}{lr}
\hline \hline Stellar parameters & \\
Stellar mass & $0.5 M_{\odot}$ \\
Stellar radius & $2 R_{\odot}$ \\
FUV luminosity & $3 \times 10^{32} \mathrm{erg} \mathrm{s}^{-1}$ \\
EUV photon number rate & $6 \times 10^{41} \mathrm{~s}^{-1}$ \\
X-ray luminosity & $10^{30} \mathrm{erg} \mathrm{s}^{-1}$ \\
\hline Gas/dust properties & \\
Species & $\mathrm{H}, \mathrm{H}^{+}, \mathrm{H}_{2}, \mathrm{H}_{2}+, \mathrm{CO}, \mathrm{O}, \mathrm{C}^{+}, \mathrm{e}^{-}$ \\
Carbon abundance & $0.927 \times 10^{-4} \times Z / Z_{\odot}$ \\
Oxygen abundance & $3.568 \times 10^{-4} \times Z / Z_{\odot}$ \\
Dust to gas mass ratio & $0.01 \times Z / Z_{\odot}$ \\
\hline
\end{tabular}

The simulations are performed in $2 \mathrm{D}$ spherical polar coordinates $(r, \theta)$. The disk is assumed to be symmetric around the rotational axis $(\theta=0)$ and to the midplane $(\theta=\pi / 2)$. The time evolution of the gas density, velocity, energy, and chemical abundances are solved.

${ }^{7}$ We refer to photons with $h \nu \geq 0.1 \mathrm{keV}$ as X-rays in the present study. 
Although the computational domain is defined in $2 \mathrm{D}$, we solve the azimuthal velocity evolution as well as the poloidal velocity $\boldsymbol{v}_{\mathrm{p}}=\left(v_{r}, v_{\theta}\right)$. In the energy equation, relevant heating/cooling sources are included (Paper I). For the chemical evolution, we take into account both the advection and chemical reactions.

$\mathrm{X}$-ray heating, X-ray ionization, and the associated chemical reactions involving $\mathrm{H}_{2}{ }^{+}$are added to our chemistry model. We describe the implementation of these physical processes in Appendix $\mathrm{A}$

The equation of state is given as in Paper I, but the ratio of specific heat $\gamma$ is calculated with considering the contribution of $\mathrm{H}_{2}{ }^{+}$,

$$
\gamma=1+\frac{y_{\mathrm{H}}+y_{\mathrm{H}^{+}}+y_{\mathrm{H}_{2}}+y_{\mathrm{H}_{2}}+y_{\mathrm{e}}}{\frac{3}{2} y_{\mathrm{H}}+\frac{3}{2} y_{\mathrm{H}^{+}}+\frac{5}{2} y_{\mathrm{H}_{2}}+\frac{5}{2} y_{\mathrm{H}_{2}}+\frac{3}{2} y_{\mathrm{e}}} .
$$

FUV, EUV, and X-ray radiative transfer is solved by ray-tracing but with neglecting scattering. The diffuse EUV due to recombination of hydrogen has been proposed as an important component to drive photoevaporation (Hollenbach et al. 1994), but a recent study by Tanaka et al. (2013) shows that the direct EUV is dominant over the diffuse one. Hollenbach et al. (1994) uses a disk model which has an infinitesimally thin disk structure in the region outer than the gravitational radius and it has a finite scale height in the region inner than the radius. Tanaka et al. (2013) uses another one where disk scale height is finite at any distance. The difference in the conclusions between these studies can be partly derived from the difference of the adopted disk models. This implies that the direct EUV can be dominant in the outer region unless there is a geometrically thick region which completely attenuates the direct EUV as in Hollenbach et al. (1994). We note that Hollenbach (2017) discusses the causes for the differences in conclusions between these two studies. Our disk model does not have the geometrically thick region. Therefore, we neglect the diffuse EUV component in our model. The FUV and EUV radiative transfer is done as in Paper $\mathrm{I}$, whereas the X-ray radiation transfer is described in Appendix $\mathrm{A}$. The dust temperature is calculated by following radiative transfer for the direct stellar irradiation component and the diffusive dust (re-)emission component, using a hybrid scheme of Kuiper et al. (2010).

We set the computational domain on $r=[1,400]$ au and $\theta=[0, \pi / 2] \mathrm{rad}$. Since the gravitational radius for a $0.5 M_{\odot}$ central star is $\sim 0.7$ au for $10^{4} \mathrm{~K}$ ionized gas (Liffman 2003), setting the inner boundary at 1 au may result in an underestimate of the photoevaporative mass loss rate. However, the contribution from within 1 au is only a small fraction of the total mass loss rate 8 Therefore, we set the inner boundary at $r=1$ au in our simulations. Hollenbach (2017) show that using a finite-size sink would result in missing the attenuation of the direct EUV inside it. This effect may reduce the direct EUV reaching the outer disk and yield a smaller EUV photoevaporation rate by a factor. Ideally, it would be better to define a computational domain which extends down to

8 In Paper I, we ran simulations with smaller inner boundaries of $0.1 \mathrm{au}, 0.35 \mathrm{au}$, and $0.5 \mathrm{au}$. We found that the density of the ionized atmosphere is too small to shield EUV photons and the EUV photons actually reach the outer region. We thus justify our setting of the inner boundary at 1 au. the stellar surface, but it is clearly beyond the limitation of the currently available numerical methods. We do not set the strict surface boundary conditions but note here that the accreting gas to the stellar surface might shield the high energy photons (Takasao et al. 2018).

We choose a sufficiently large domain to avoid spurious reflection of soundwaves and gas flows. In Paper I, we show that a sufficiently large outer boundary can eliminate the spurious effect.

We run a set of simulations where all of the photoionization heating by EUV (hereafter, EUV heating), photoelectric heating by FUV (hereafter, FUV heating), and $\mathrm{X}$-ray heating are taken into account. In order to isolate the effects of X-ray heating, we also run simulations without FUV heating. The resulting photoevaporation rates are compared with the results of Paper I, where X-rays are not included. Hereafter, we label the sets of our simulations according to which (or the combination) of FUV, EUV, and X-ray heating is taken into account. A simulation is labeled as "Run YYY", where "YYY" specifies which of the photo-heating sources are included. For example, in "Run FEX", all the photo-heating effects are included If only EUV heating is taken into account, the simulation is referred to as "Run E". In addition, when we refer to a simulation with metallicity $Z=10^{C} Z \odot$, we append " $/ Z C$ " to the above labels. For instance, when we refer to the simulation with $Z=10^{-0.5} Z_{\odot}$ in which FUV and EUV heating are taken into account, we refer to the simulation as "Run FE/Z-0.5".

\section{RESULTS}

In this section, we study the photoevaporation rate and the structure of PPDs. We first present the results of the solar metallicity case in Section 3.1] We then discuss the metallicity dependence in Section 3.2 and Section 3.3

\subsection{Solar Metallicity Disks}

FIG. [1 shows that photoevaporative flows are excited in all the cases. Dense neutral photoevaporative flows composed of atomic and molecular hydrogen are driven in Run FEX/Z0 and Run FE/Z0, but not in Run EX/Z0. Ionized photoevaporative flows, which consist of ionized hydrogen, are driven in all the three simulations. FUV heating is an important process to drive the neutral flows. $\mathrm{X}$-ray heating is ineffective to drive photoevaporation in Run EX/Z0, where EUV-driven flows are excited only from the low density, disk surface regions.

Following Paper I, we measure the photoevaporation rate $\dot{M}_{\mathrm{ph}}$ by integrating the mass flux normal to a spherical surface $S$

$$
\dot{M}_{\mathrm{ph}}=\int_{S} d \boldsymbol{S} \cdot \rho \boldsymbol{v}=r_{S}^{2} \int_{S} d \theta d \phi \sin \theta \rho v_{r},
$$

where $d \boldsymbol{S}$ is an integral element vector of the surface and $r_{S}$ is the radius of the sphere. A gas parcel is regarded as unbound, and its contribution to $\dot{M}_{\mathrm{ph}}$ is counted only if the total specific enthalpy of the gas

$$
\eta=\frac{1}{2} \boldsymbol{v}^{2}+\frac{\gamma}{\gamma-1} c_{s}^{2}-\frac{G M_{*}}{r},
$$

is positive at the surface (Liffman 2003). With this condition, contributions from the bound disk region $(\eta<0)$ to $\dot{M}_{\text {ph }}$ are effectively excluded. 


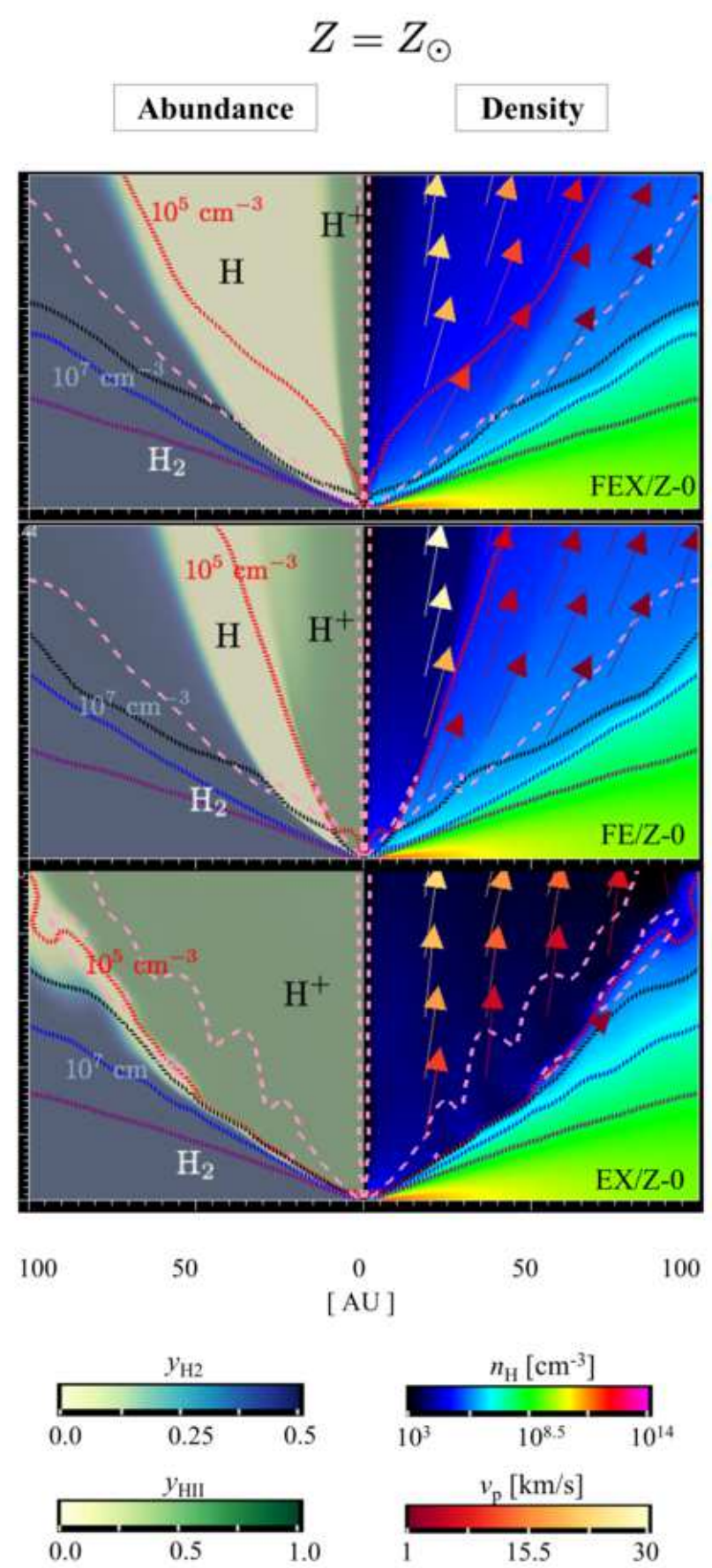

FIG. 1. - Solar-metallicity disk structures in Run FEX/Z0 (top), Run FE/Z0 (middle), and Run EX/Z0 (bottom). The left panels show the chemical structures and the right panels show the density structures. The arrows indicate the velocity fields with the poloidal velocities of $v_{\mathrm{p}}>1 \mathrm{~km} \mathrm{~s}^{-1}$. The dotted lines are the density contours of $n_{\mathrm{H}}=10^{5} \mathrm{~cm}^{-3}$ (red), $n_{\mathrm{H}}=10^{6} \mathrm{~cm}^{-3}$ (black), $n_{\mathrm{H}}=10^{7} \mathrm{~cm}^{-3}$ (blue), and $n_{\mathrm{H}}=10^{8} \mathrm{~cm}^{-3}$ (purple). The pink dashed lines indicate the sonic surface.

In Section 3.3 of Paper I, we show that photoevaporation rates measured as in Eq. (2) generally increase with $r_{S}$. This is because the temperatures in the launching regions of photoevaporative flows, also called base temperatures, are generally larger than necessary to escape from the gravity in the outer region beyond the gravitational radius; There are contributions to the mass loss in the outer region. Thus, photoevaporation rates should be estimated with different $r_{S}$ to count the contributions. We calculate $\dot{M}_{\mathrm{ph}}$ with setting $r_{S}=20 \mathrm{au}, 100 \mathrm{au}, 200 \mathrm{au}$ for each of Run FEX, Run FE, and Run EX. For the solar metallicity disk, we also perform Run X/Z0 to examine explicitly the contribution of X-ray-driven flows to $\dot{M}_{\mathrm{ph}}$. The photoevaporation rates with $r_{S}=20$ au include contribution from the inner part, while those with $r_{S}=100 \mathrm{au}$ and $r_{S}=200 \mathrm{au}$ include contribution from the whole region of a disk. Generally, in our simulations, photoevaporation rates vary in time for the first $\sim 5000$ years, but then converge afterward. We take a time-averaged photoevaporation rate over 5000 years. The resulting photoevaporation rates for Run FEX/Z0, Run FE/Z0, Run EX/Z0, and Run X/Z0 are given in TABLE 2 for each case of $r_{S}=20 \mathrm{au}, r_{S}=100 \mathrm{au}$, and $r_{S}=200 \mathrm{au}$, and are also plotted in FIG. 3 .

TABLE 2

Resulting Photoevaporation RATES $\dot{M}_{\mathrm{PH}}$ OF THE SOlAR METALLICITY DISK IN $M_{\odot} \mathrm{yr}^{-1}$

\begin{tabular}{c|cccc}
$r_{S}$ & Run FEX/Z0 & Run FE/Z0 & Run EX/Z0 & Run X/Z0 \\
\hline \hline $20 \mathrm{au}$ & $6 \times 10^{-9}$ & $3 \times 10^{-9}$ & $2 \times 10^{-10}$ & $5 \times 10^{-12}$ \\
$100 \mathrm{au}$ & $2 \times 10^{-8}$ & $2 \times 10^{-8}$ & $1 \times 10^{-9}$ & $1 \times 10^{-11}$ \\
$200 \mathrm{au}$ & $3 \times 10^{-8}$ & $3 \times 10^{-8}$ & $2 \times 10^{-9}$ & $2 \times 10^{-11}$ \\
\hline
\end{tabular}

We note that $\dot{M}_{\mathrm{ph}}$ in Run FEX/Z0 is slightly higher than in Run FE/Z0. Also, $\dot{M}_{\mathrm{ph}}$ in Run EX/Z0 is about an order of magnitude smaller than in Run FEX/Z0 and in Run FE/Z0. We find a very small $\dot{M}_{\text {ph }}$ in Run X/Z0. Overall, these results suggest that FUV is a crucial radiation component to produce a high $\dot{M}_{\mathrm{ph}}$, whereas X-rays give a minor contribution to $\dot{M}_{\mathrm{ph}}$, although X-rays affect the structure of the neutral flows (see Run FEX/Z0 and Run FE/Z0 in FIG. (1).

The X-ray heating rate in Run EX/Z0 is smaller than the FUV heating rate in Run FEX/Z0 and in Run FE/Z0 (see the second row of FIG. (2). The neutral regions in the disk are not heated by X-rays to sufficiently high temperatures to escape from the star-disk system. The gas temperatures are nearly coupled with the dust temperatures in this region (the top right panel of FIG. 2). We conclude that X-ray heating itself is not efficient to excite photoevaporative flows. Only the EUV-driven, ionized flows contribute to the mass loss in Run EX/Z0. FIG. 1 shows that the ionized flows have densities typically several orders of magnitude smaller than the neutral flows. The resulting $\dot{M}_{\mathrm{ph}}$ of Run EX/Z0 is much smaller than those of Run FEX/Z0 or Run FE/Z0, where FUV-driven neutral flows give a large contribution to $\dot{M}_{\text {ph }}$.

FUV heating is dominant in the neutral regions in Run FEX/Z0 and Run FE/Z0 (FIG. 21). By studying these runs in detail, we find that the FUV heating rate is higher in Run FEX/Z0 than in Run FE/Z0 especially in the regions close to the ionization front. There, the gas is weakly ionized by X-rays. With the electron abundance slightly increased, dust grains recombine, the positive charges of dust grains are reduced, and then the photo- 


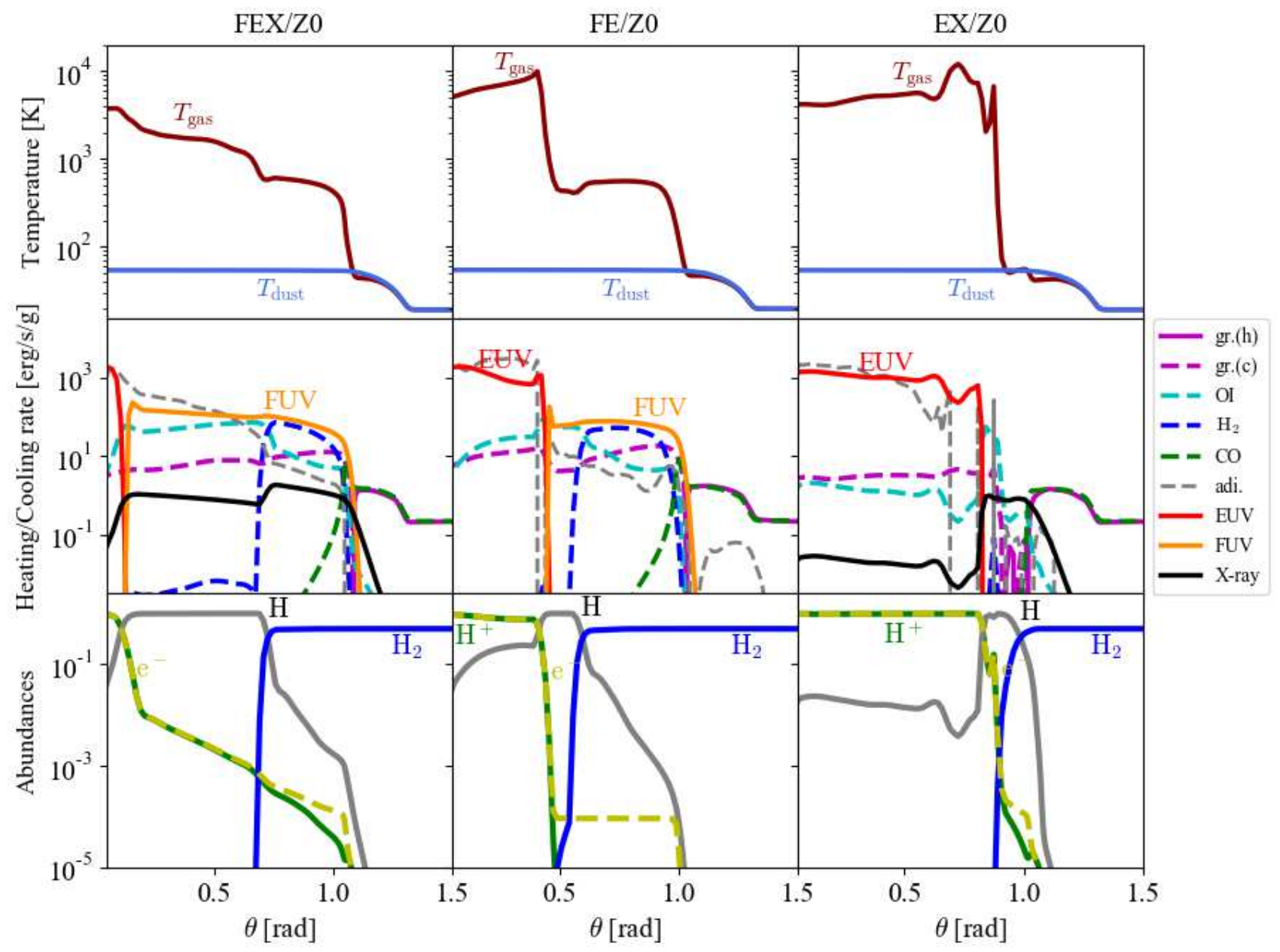

Fig. 2.- Meridional distributions of the physical quantities at $r=100$ au: gas and dust temperatures (top), specific heating/cooling rates (middle), and chemical abundances of $\mathrm{H}_{1} \mathrm{H}^{+}, \mathrm{H}_{2}$, and electrons (bottom). In the middle row, the solid lines show the heating rates of EUV (red), FUV (orange), X-ray (black), and dust-gas collision (purple), while the dashed lines show the cooling rates of dust-gas collision (purple), OI (cyan), $\mathrm{H}_{2}$ (blue), $\mathrm{CO}$ (green), and adiabatic cooling (gray). Each column shows the physical quantities of Run FEX/Z0, Run $\mathrm{FE} / \mathrm{Z} 0$, and Run EX/Z0 for solar metallicity disks from the left to the right.

electric effect efficiency is increased (Gorti \& Hollenbach 2009).

To summarize, X-ray ionization effectively strengthens FUV heating in neutral regions in a disk by increasing the photoelectric effect efficiency. Owing to the strengthened FUV heating, the temperatures of the neutral regions are higher in Run FEX/Z0 than in Run FE/Z0. With the combined FUV+X-ray heating effect, the neutral gas near the central star evaporates in Run FEX/Z0. We have checked and found that there is a large difference in $\dot{M}_{\text {ph }}$ measured with $r_{S}=20$ au between Run FEX/Z0 and Run FE/Z0 (TABLE 2). However, in these runs, the photoevaporative flows excited in the inner regions accounts for only a small fraction of the total mass loss rate, and a dominant contribution comes from outer disk regions. Therefore, there is a small difference in $\dot{M}_{\text {ph }}$ between Run FEX/Z0 and Run FE/Z0, when measured with $r_{S}=100 \mathrm{au}$ or $r_{S}=200 \mathrm{au}$.

\subsection{FUV Heating in Low Metallicity Disks}

FUV radiation can reach the deeper interior of a disk with a lower metallicity because the amount of dust grains and hence its opacity are correspondingly smaller. This results in exciting photoevaporative flows with higher densities. On the other hand, the FUV heating becomes progressively inefficient at low metallicities compared to dust-gas collisional cooling. The relatively inefficient FUV heating lowers the base temperatures, and FUV-driven flows are not excited in the "cool" disk. Consequently, $\dot{M}_{\text {ph }}$ of Run FE increases in the range of $10^{-1} Z_{\odot} \lesssim Z \lesssim 10^{0.5} Z_{\odot}$ but decreases in the range of $10^{-2} Z_{\odot} \lesssim Z \lesssim 10^{-1} Z_{\odot}$ (see FIG. [3). Note that a major contribution to the mass loss rate comes from FUV-driven photoevaporative flows in runs with $Z \gtrsim 10^{-2} Z_{\odot}$. EUV-driven flows are important in runs with $Z \lesssim 10^{-2} Z_{\odot}$, where the metallicity dependence is small. 

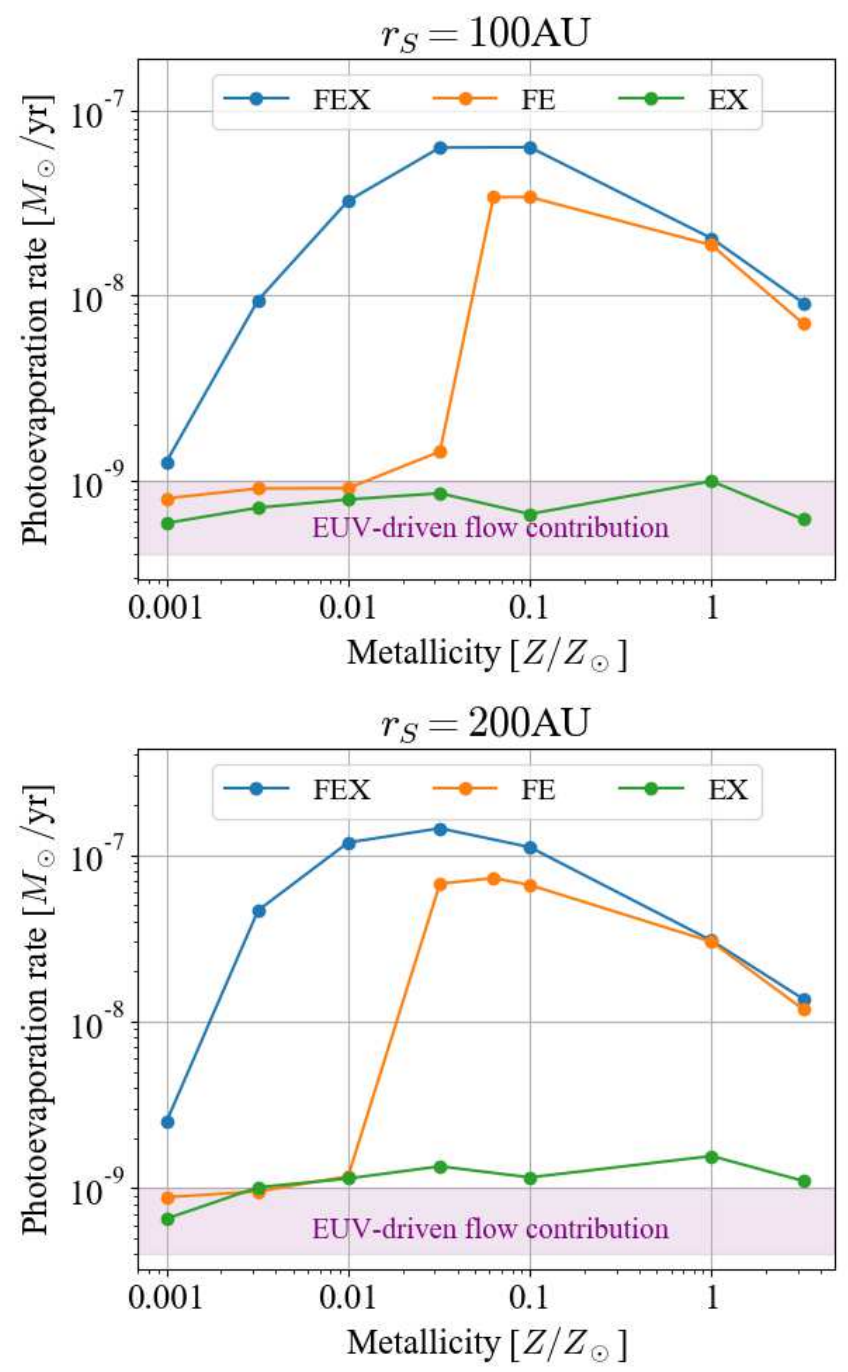

FIG. 3.- Each panel shows the difference in the metallicity dependences of the photoevaporation rates for Run FEX (blue), Run FE (orange), and EX (green). The purple regions show approximate EUV-driven flow contributions $\left(\dot{M}_{\mathrm{EUV}} \simeq 0.4-1 \times\right.$ $10^{-9} M_{\odot} \mathrm{yr}^{-1}$ ), separating the EUV photoevaporation rates from the total photoevaporation rates. The photoevaporation rates are calculated with $r_{S}=100 \mathrm{au}$ and $r_{S}=200 \mathrm{au}$ in the top and bottom panels, respectively.

Although including X-rays affects the metallicity dependence of $\dot{M}_{\mathrm{ph}}$ (compare Run FEX and Run FE in FIG. 3), the overall trend appears quite similar; $\dot{M}_{\mathrm{ph}}$ of Run FEX increases as metallicity decreases in $Z \gtrsim$ $10^{-1.5} Z_{\odot}$ because of the reduced opacity effect, while $\dot{M}_{\text {ph }}$ decreases with metallicity in $Z \lesssim 10^{-1.5} Z_{\odot}$ because FUV photoelectric heating becomes less effective than dust-gas collisional cooling.

\subsection{The Effect of X-ray Ionization}

There is a significant difference between Run FEX and Run FE at very low metallicities of $Z \lesssim 10^{-1.5} Z_{\odot}$. In Run FEX, X-ray ionization raises the electron abundance in the neutral region of the disk, and effectively strengthens the FUV heating. The gas temperature in the neutral region is higher than in Run FE. This causes the neutral gas to evaporate from even closer regions to the star. However, with the low metallicities of $Z \gtrsim 10^{-1.5} Z_{\odot}$, the contribution from the inner region is still small with respect to the total photoevaporation rate (FIG. 33). This is similar to the case with solar metallicity as discussed in Section 3.1. In other words, FUV heating can drive neutral photoevaporative flows even without the strengthening effect of X-rays in this metallicity range; the photoevaporation rates of Run FEX and Run FE are close to each other. This is in good contrast to the runs with $10^{-2.5} Z_{\odot} \lesssim Z \lesssim 10^{-1.5} Z_{\odot}$.

In the range of $Z \lesssim 10^{-1.5} Z_{\odot}, \dot{M}_{\mathrm{ph}}$ of Run FEX decreases with metallicity because of the efficient dust-gas collisional cooling relative to FUV heating, but we find a more gradual decline of $\dot{M}_{\mathrm{ph}}$ than in Run FE. In Run FEX, the electron abundance in the neutral region is increased by the effect of X-rays. Hydrogen is the dominant X-ray absorber, and thus the electron abundance in the neutral region is essentially independent of metallicity (FIG. 4). Since the photoelectric effect efficiency

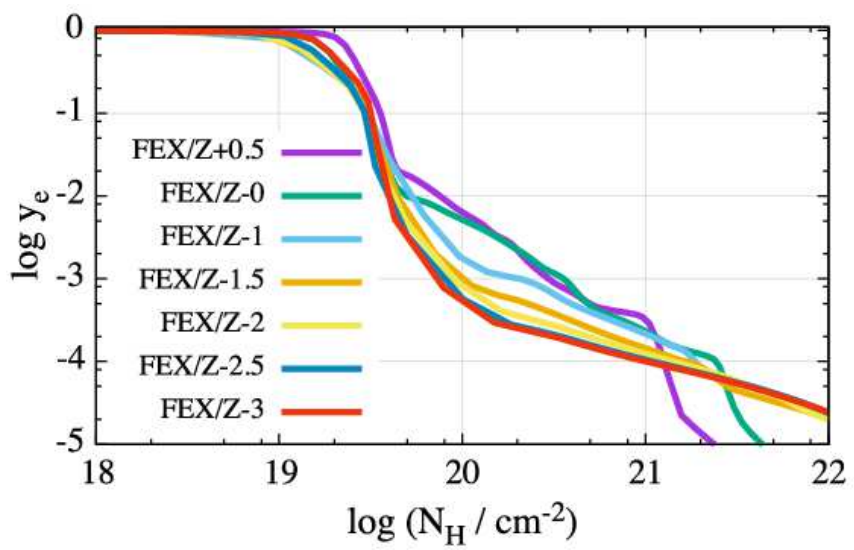

Fig. 4.- Meridional distributions of electron abundance at $r=$ $100 \mathrm{au}$ for various metallicity disks. All of the data are taken from Run FEX. Note that we here use hydrogen nuclei column density $N_{\mathrm{H}}$ instead of $\theta$ unlike in FIG. 2

depends on electron density only through the ratio of the dust/PAH photoionization rate to the dust/PAH recombination rate $\gamma_{\mathrm{pe}}=G_{\mathrm{FUV}} \sqrt{T} / n_{\mathrm{e}}$ (see Eq.[41] of Paper I for details), it is not affected by metallicity, at least explicitly, when the electron abundance is largely set by hydrogen ionization. As a result, FUV heating remains effective even at low metallicities $\left(Z \lesssim 10^{-1.5} Z_{\odot}\right)$ in Run FEX than in Run FE, and thus $\dot{M}_{\text {ph }}$ in Run FEX decreases more gradually.

We find a large difference in $\dot{M}_{\mathrm{ph}}$ between Run FEX and Run FE, especially in the range $10^{-2.5} Z_{\odot} \lesssim Z \lesssim$ $10^{-1.5} Z_{\odot}$. This can be attributed to the effect of the $\mathrm{X}$-ray radiation through partial ionization. For example, in the disk with $Z=10^{-2} Z_{\odot}$, the electron abundance in Run FEX/Z-2 is about two orders of magnitude larger than in Run FE/Z-2 in the low density part $\left(n_{\mathrm{H}} \sim 10^{5}-10^{6} \mathrm{~cm}^{-3}\right)$ at around $100 \mathrm{au}$. Correspondingly, the ratio of the dust photoionization rate to the dust recombination rate $\gamma_{\mathrm{pe}}=G_{\mathrm{FUV}} \sqrt{T} / n_{\mathrm{e}}$ (cf. Appendix A of Paper I) is small, with the typical value of $\sim 10^{3}\left(n_{\mathrm{e}} / 100 \mathrm{~cm}^{-3}\right)^{-1}$ in the low-density region. This is about two orders of magnitude smaller than in Run 
FE/Z-2. Therefore, the photoelectric effect efficiency

$$
\begin{aligned}
\epsilon_{\mathrm{pe}} & =\left[\frac{4.87 \times 10^{-2}}{1+4 \times 10^{-3} \gamma_{\mathrm{pe}}^{0.73}}\right. \\
& \left.+\frac{3.65 \times 10^{-2}\left(T / 10^{4} \mathrm{~K}\right)^{0.7}}{1+2 \times 10^{-4} \gamma_{\mathrm{pe}}}\right]
\end{aligned}
$$

is larger by about an order of magnitude (Bakes \& Tielens 1994). The temperature is increased by a factor of a few, and then the gas satisfies the enthalpy condition $\eta>0$. X-rays also affect other regions of the disk in a similar manner; the total specific enthalpy of the gas is increased to be positive. FIG. 5 shows that the neutral region of Run FEX/Z-2 partly satisfies $\eta>0$, whereas the region with $\eta>0$ appears to overlap with the $\mathrm{H}$ II region in Run FE/Z-2. In runs with $Z=10^{-2} Z_{\odot}$, incorporating $\mathrm{X}$-ray ionization results in driving neutral photoevaporative flows, which significantly contribute to the mass loss rate. Without $\mathrm{X}$-rays, however, the neutral gas flows are not excited, and only EUV-driven ionized gas flows contribute to the mass loss. Consequently, Run FEX/Z-2 shows a significantly larger $\dot{M}_{\mathrm{ph}}$ than Run FE/Z-2. The same conclusion holds for Run FEX and Run FE with metallicities in the range of $10^{-2.5} Z_{\odot} \lesssim Z \lesssim 10^{-1.5} Z_{\odot}$.

In the very low metallicity of $Z \underset{\sim}{\approx} 10^{-3} Z_{\odot}$, even though FUV heating is strengthened by the X-ray ionization effect, neutral flows are not excited because dustgas collisional cooling becomes more efficient than FUV heating. Therefore, there is not a significant difference between the photoevaporation rates of Run FEX/Z-3 and Run FE/Z-3.

\subsection{PPD Lifetime}

Regarding metallicity dependence of PPD lifetimes, it is suggested that typical lifetimes of protoplanetary disks are $3 \mathrm{Myr}$ for solar metallicity disks and $1 \mathrm{Myr}$ for those with $Z=0.2 Z_{\odot}$ (Yasui et al. 2009, 2010, 2016b,a). This metallicity dependence of the lifetimes can be fit as $T_{\text {life }} \propto Z^{0.7}$. In the present study, the resulting photoevaporation rate of Run FEX has metallicity dependences of $\dot{M}_{\mathrm{ph}} \propto Z^{-0.6}$ for $r_{S}=200 \mathrm{au}$, in $0.1 Z_{\odot} \leq Z \leq$ $10^{0.5} Z_{\odot}$, while in Run FE $\dot{M}_{\mathrm{ph}} \propto Z^{-0.4}$. These metallicity dependences are consistent with the observational metallicity dependence of the lifetimes because disk lifetimes are approximately calculated as $T_{\text {life }} \propto \dot{M}_{\mathrm{ph}}^{-2 / 3}$ (Ercolano \& Clarke 2010). Since X-ray radiation itself does not excite photoevaporative flows in a direct manner, the photoevaporation rate of Run EX is largely contributed by the EUV-driven flows. Therefore, $\dot{M}_{\mathrm{ph}}$ is generally metallicity-independent and is significantly smaller than in Run FEX or Run FE, where FUV-driven flows contribute to the mass loss. This suggests that in the case EUV heating mainly contributes to photoevaporation, EUV and X-ray radiation does not cause metallicity dependence in PPD lifetimes. Hence, if the metallicity dependence of the lifetimes is originated from the metallicity dependence of photoevaporation, our model indicates that FUV photoevaporation has a major effect on the disk lifetimes.

\section{DISCUSSION}

Our conclusion regarding the effects of X-ray radiation is qualitatively consistent with that of Gorti \& Hollenbach (2009) (hereafter, GH09), who conclude that X-ray photoionization increases the efficiency of photoelectric heating and enhance the FUV photoevaporation rate. Although X-ray heating has been proposed as an important cause of photoevaporation in several studies (Ercolano et al. 2008, 2009; Owen et al. 2012), our direct comparison shows that X-rays alone do not drive strong photoevaporation, in agreement with the conclusions of Alexander et al. (2004), Gorti \& Hollenbach (2009), and Wang \& Goodman (2017). In the following, we discuss the effect of a few elements associated with our X-ray radiation model.

\subsection{X-Ray Spectral Hardness}

The hardness of the adopted X-ray spectrum affects the strength of X-ray photoevaporation. Table 1 of Ercolano et al. (2009) shows that the photoevaporation rate decreases with the "pre-screening" column, i.e., the hardness of the incident flux on a disk. With the prescreening column of $N_{\mathrm{H}} \geq 10^{21} \mathrm{~cm}^{-2}$, there are virtually no photons with $\lesssim 0.1 \mathrm{keV}$ reaching the interior of a disk (see Figure 3 of Ercolano et al. 2009). In this case, the resulting photoevaporation rate is of the order of $10^{-11} M_{\odot} \mathrm{yr}^{-1}$, and is smaller by two orders of magnitude than the case with the pre-screening column of $N_{\mathrm{H}} \leq 10^{20} \mathrm{~cm}^{-2}$, where the EUV component $(\leq 0.1 \mathrm{keV})$ also heats the disk. The result suggests that using a hard X-ray spectrum results in inefficient X-ray photoevaporation, as has been also pointed out by Gorti et al. (2015). The X-ray spectrum used in the present study is similar to that with pre-screening column of $N_{\mathrm{H}} \sim$ $10^{21} \mathrm{~cm}^{-2}$ with which the X-ray photoevaporation rate is small $\left(\sim 10^{-11} M_{\odot} \mathrm{yr}^{-1}\right)$ (Ercolano et al. 2009).

In order to examine whether using a softer spectrum affects the resulting $\mathrm{X}$-ray photoevaporation rate, we additionally perform test simulations where our fiducial X-ray spectrum is shifted to lower energies. We shift the fiducial X-ray spectrum $F(E)$ as $F(E \times \sqrt{10})$ and $F(E \times 10)$ while fixing the total luminosity of $10^{30} \mathrm{erg} \mathrm{s}^{-1}$. The shifted spectra are shown by the red and green lines in FIG. 6. Hereafter, we refer to the shifted spectrum colored in green as the soft spectrum, and the other colored in red as the intermediate spectrum. The photoheating rates are calculated by Eq. (A14) as in our fiducial model. For the heating efficiency, we use the same $f_{h}$ given by Eq. A15. Although $f_{h}$ might be larger for the softer spectra because all the primary electron energy goes into heat through Coulomb interactions with the ambient electrons when $y_{\mathrm{e}} \sim 1$ (Maloney et al. 1996), we do not model the heating efficiency as a function of photon energy. Instead, in Section 4.2. we study simulations with $f_{h}=1$, corresponding to the limiting case where all the absorbed energy goes into heating. FUV heating is not taken into account in our test simulations presented here.

FIG. 7 shows that the maximum values of the photoheating rates (the black lines) are larger by about an order of magnitude for the soft and intermediate spectra than that for our fiducial spectrum which is shown in the right column of FIG. 2. The specific photo-heating 


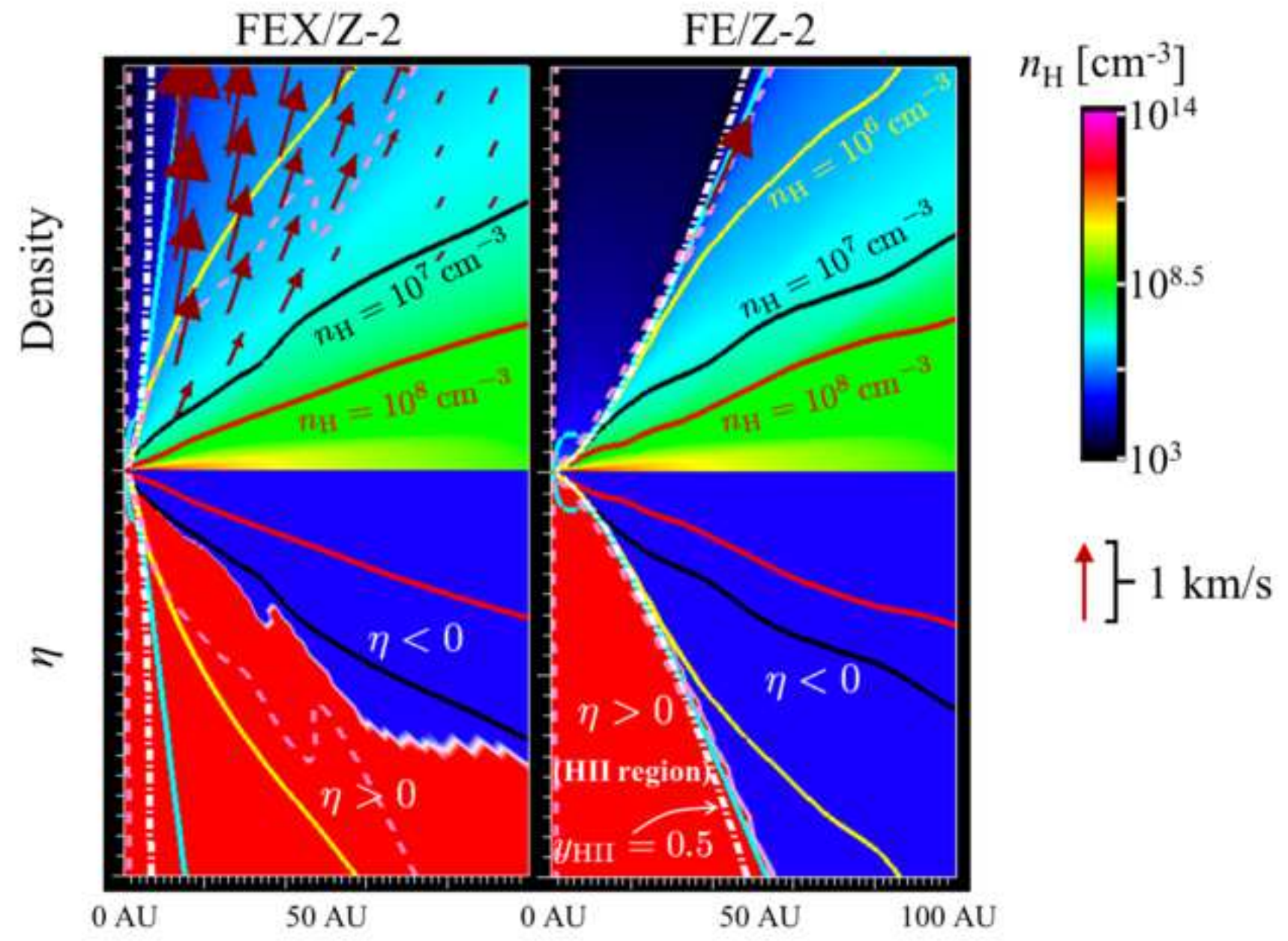

FIG. 5.- Snapshots of FEX/Z-2 (left) and FE/Z-2 (right). The top panels show the density distributions, and the bottom panels show the distributions of $\eta$ (Eq. (3) ) where the red and blue regions indicate $\eta>0$ (unbound) regions and $\eta<0$ (bound) regions, respectively. The arrows show the velocity with $0.25 \mathrm{~km} \mathrm{~s}^{-1}<v_{\mathrm{p}}<0.5 \mathrm{~km} \mathrm{~s}^{-1}$ and they are scaled by its magnitude. The velocity field in the $\mathrm{H}$ II regions $\left(y_{\mathrm{HII}}>0.5\right)$ are not shown for clarity. The solid lines show density contours of $n_{\mathrm{H}}=10^{5} \mathrm{~cm}^{-3}(\mathrm{cyan}), n_{\mathrm{H}}=10^{6} \mathrm{~cm}^{-3}$ (yellow), $n_{\mathrm{H}}=10^{7} \mathrm{~cm}^{-3}$ (black), and $n_{\mathrm{H}}=10^{8} \mathrm{~cm}^{-3}$ (red). Note that the velocity arrows and density contours are drawn in different manners from FIG. 1 The white dot-dashed lines show contours of $y_{\mathrm{HII}}=0.5$, which is a rough boundary between the H II region and the other. The pink dashed lines indicate the sonic surface.

rate is smaller for higher energy photons. FIG. 7 also shows that low energy photons are nearly completely absorbed in the region close to the ionization front. Since the cross section of the disk medium is larger for lower energy photons, adopting a softer spectrum results in a higher specific photo-heating rate, but the low energy photons are absorbed in regions with small gas densities.

The photo-heating raises the gas temperature only in the region close to the ionization front, whereas a large part of the neutral region remains at relatively low temperatures, as seen in FIG. 17. The gas is not hot enough to launch neutral outflows, and the EUV-driven, ionized flows dominantly contribute to mass loss rate. FIG. 8 compares the photoevaporation rates for the soft, intermediate, and fiducial spectra. Clearly, in our test simulations, the spectral hardness does not critically affect the photoevaporation rate, although it changes the thermal and chemical structure of the disk (Fig. 7).

\subsection{Effect of the Heating Efficiency $f_{h}$}

Another important factor is the heating efficiency $f_{h}$. Adopting $f_{h}=1$ raises the photo-heating rate. We see in FIG. 7 that the neutral region has a higher temperature when using $f_{h}=1$ (the right column) than when using $f_{h}$ of Eq. A15 (the left and middle columns). Consequently, the photoevaporation rate with the intermediate spectrum with $f_{h}=1$ are the highest (FIG. 8). Note that the cross-section-weighted mean energy $\bar{E}$ is $0.03 \mathrm{keV}$ and $0.11 \mathrm{keV}$ for the soft and intermediate spectra, respectively. For our fiducial spectrum, $\bar{E}=0.34 \mathrm{keV}, f_{h}=1$ does not significantly change the photoevaporation rate. We conclude that high energy photons with $\gtrsim 0.1 \mathrm{keV}$ are ineffective to heat the neutral gas and do not excite dense photoevaporative flows. The same is true for the soft and intermediate spectra. Our test simulations show that the effective component for photoevaporative mass loss is not X-ray $(0.1 \mathrm{keV} \leq h \nu \leq 10 \mathrm{keV})$, but EUV $(13.6 \mathrm{eV} \leq h \nu \leq 100 \mathrm{eV})$. Hard EUV photons with $\sim 10^{2} \mathrm{eV}$ most efficiently drive disk mass loss.

FIG. 8 compares directly $\dot{M}_{\text {ph }}$ in the above test runs 


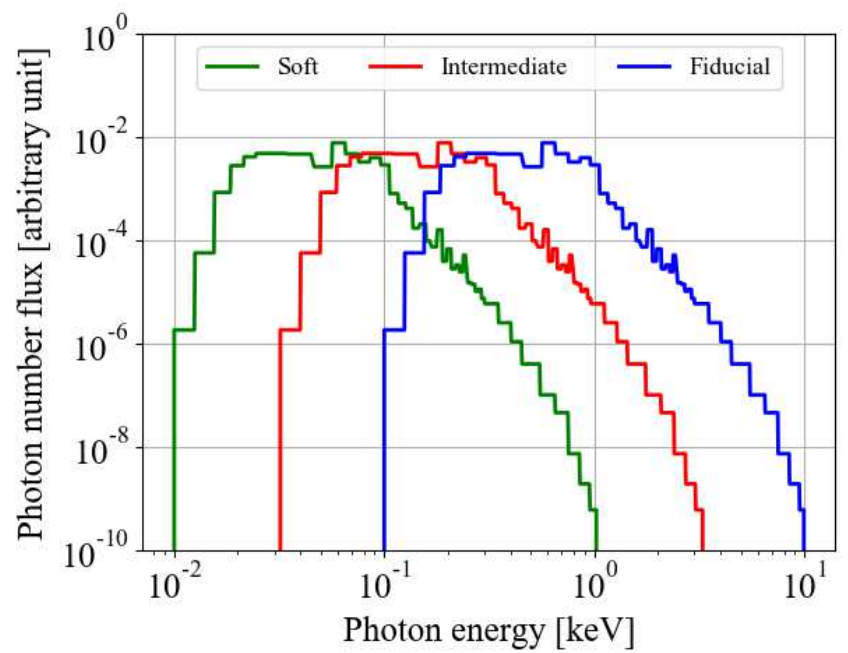

FIG. 6.- Fiducial SED (blue) and logarithmically shifted SEDs (red and green) for X-rays. The shifted SEDs are given as $F(E \times$ $\sqrt{10})$ (red) and $F(E \times 10)$ (green), where $F(E)$ is the fiducial SED function.

with that of Run X/Z0. Again, we confirm that X-rays are ineffective to excite photoevaporative flows. When FUV heating is absent (a somewhat artificial condition, but for the purpose of direct comparison), EUV mainly contributes to the photoevaporative mass loss. This is consistent with Table 1 of Ercolano et al. (2009), where we find the photoevaporation rates are significantly low when the EUV component is screened out (see also Gorti et al. (2015)).

In Ercolano et al. (2009), the EUV component reaches the disk surface if the pre-screening column density $N_{\mathrm{H}}$ is smaller than $10^{20} \mathrm{~cm}^{-2}$. The spectrum model of Ercolano et al. (2009) with $N_{\mathrm{H}}=10^{19}-10^{20} \mathrm{~cm}^{-2}$ is similar to our intermediate spectrum. Their resulting photoevaporation rate of Ercolano et al. (2009) is $\dot{M}_{\mathrm{ph}} \simeq 4 \times 10^{-9} M_{\odot} \mathrm{yr}^{-1}$, which is close to $\dot{M}_{\mathrm{ph}}$ of the test simulation with the intermediate spectrum and $f_{h}=1$ (FIG. 8). Further, the photoevaporation rate differs only by a factor of two from that of Owen et al. (2012), where an "ionization parameter" approach is used with the unscreened spectrum of Ercolano et al. (2009) to calculate gas temperatures in the hydrodynamics simulations. The agreement between the photoevaporation rates in Owen et al. (2012) and the present study implies that, for a spectrum with a large amount of $\sim 0.1 \mathrm{keV}$ photons, the ionization parameter approach yields essentially the same results as those derived with heating and radiative cooling in a consistent manner. Nonetheless, self-consistent calculations such as ours are necessary to investigate the relative importance between X-ray and FUV photons.

The opening angle of the $\mathrm{H}$ II region is narrower and the resulting photoevaporation rate is larger by about a factor of two in Owen et al. (2012) than those in our intermediate $\mathrm{EX} / \mathrm{Z} 0$ with $f_{h}=1$ (the right column in FIG. 7). Since these differences likely originate from a number of differences in the adopted methods, it would be difficult to specify the causes. Nevertheless, the results of our intermediate $\mathrm{EX} / \mathrm{Z0}$ with $f_{h}=1$ and with the fiducial $f_{h}(\mathrm{Eq}$. A15) provide important clues, because the different $f_{h}$ results in different opening angles and photoevaporation rates (FIG. 7) and FIG. 8). The results suggest that a higher heating efficiency $f_{h}$ tends to generate a narrower H II region and a larger photoevaporation rate. Thus, we expect that heating efficiency is higher in Owen et al. (2012) than ours. Actually, the temperature is typically $\sim 4000-5000 \mathrm{~K}$ in the $2-10$ au region in Owen et al. (2012), while it is $2000-4000 \mathrm{~K}$ in our intermediate $\mathrm{EX} / \mathrm{Z0}$ with $f_{h}=1$. Hence, we conclude that the narrower opening angle in Owen et al. (2012) may be attributed to a larger heating efficiency. The high heating efficiency could be realized when the ionization degree is large owing to more efficient Coulomb interactions between the ejected electron and the ambient electrons. We do not account for the effect in our simulations. This effect is neglected in the present study for simplicity. Incorporating the effect can yield a larger heating efficiency (Shull \& van Steenberg 1985), and Xray heating rates could be increased especially in the region where the ionization degree is high. On the other hand, since a highly ionized medium hardly absorbs Xrays, the heating rate can be also lowered in such regions. With the electron abundances in the neutral regions of our model $y_{\mathrm{e}} \sim 10^{-4}-10^{-2}$ (FIG. 2 and FIG. 7), taking account of the dependence on the electron abundance could increase X-ray heating rates by a small factor in the neutral regions. We note that a higher heating rate also results in a narrower $\mathrm{H}$ II region in Run FEX and Run FE, where the opening angle is smaller when FUV heating is strengthened by X-ray (FIG. 1 and FIG. 5).

Wang \& Goodman (2017) show that disabling efficient cooling processes results in large photoevaporation rates due to hard X-rays with $1 \mathrm{keV}$. In order to examine if this is also the case in the fiducial model of the present study, we further perform a test simulation, where $f_{h}=1$ and the same thermal processes as Run X/Z0 are used except that all of the line cooling are disabled. The resulting photoevaporation rate is $5 \times 10^{-9} M_{\odot} \mathrm{yr}^{-1}$, which is modestly larger than our EUV photoevaporation rates. Thus, if all of the primary electron energy go into heating and line cooling processes are not effective, which might be unrealistic, hard X-rays can also cause a relatively efficient mass loss.

Overall, the effectiveness of X-rays on driving photoevaporation significantly depends on the heating efficiency $f_{h}$ and the exact shape of the spectrum of high energy photons especially around $\sim 0.1 \mathrm{keV}$. Hence, for a comprehensive study of X-ray photoevaporation, it is necessary to model the heating efficiency in a consistent manner, and to adopt a realistic spectrum.

Finally, we note that the photoevaporation rate likely depends on metallicity when assuming a hard EUV spectrum. Since the efficiency of radiative cooling due to metals in the neutral region, such as $\mathrm{O}$ I cooling and dust-gas collisional cooling, decreases with metallicity, there may be metallicity dependence of EUV/X-ray photoevaporation when the exact spectral shape is taken into account. Similarly, different FUV spectra should also result in a different photoevaporation rate. Further studies are warranted to address these issues associated with detailed conditions.

\subsection{Uncertainties in Input Parameters}




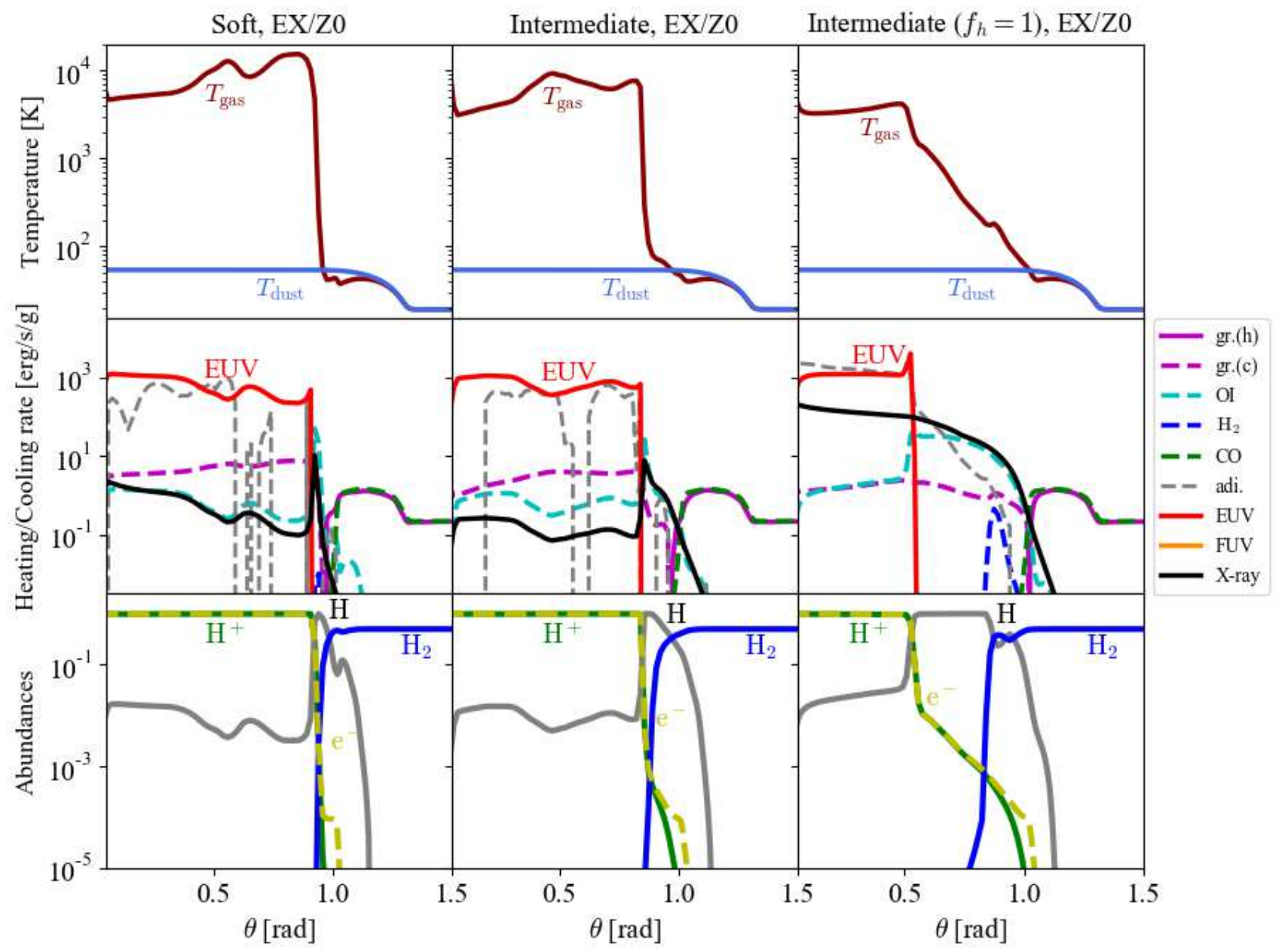

FIG. 7. - Meridional distributions of the physical quantities at $r=100$ au in the simulations, where the soft spectrum (left), intermediate spectrum (middle), and intermediate spectrum with using $f_{h}=1$ (right) are used. The panels are shown in the same manner as FIG. 2 Note that though the soft and intermediate spectra technically contain the EUV component $(13.6 \mathrm{eV} \leq h \nu \leq 100 \mathrm{eV})$, we refer to the photo-heating calculated with using the spectra as X-ray heating.

We have concluded that FUV effectively drives dense neutral flows, while X-ray is ineffective. The conclusion should depend on input parameters such as the abundance of polycyclic aromatic hydrocarbons (PAHs) and luminosities/spectra of FUV/X-ray.

PAHs and very small grains significantly contribute to photoelectric heating, and thus their abundances affect FUV photoevaporation rates (Gorti \& Hollenbach 2008; Gorti et al. 2009, 2015; Nakatani et al. 2018). Recent observations suggest that the PAH abundances around $\mathrm{T}$ Tauri stars might be smaller than the interstellar value, which we adopt in our fiducial model, although there are uncertainties in the observational results (Geers et al. 2007; Oliveira et al. 2010; Vicente et al. 2013). In Paper $\mathrm{I}$, we investigate the effect of the reduced $\mathrm{PAH}$ abundance on FUV photoevaporation. We find that the net effect of the reduced $\mathrm{PAH}$ abundance is weakening photoelectric heating, but FUV driven flows are excited anyway even without the PAH contribution. The resulting mass loss rates are the same orders of magnitude as those with the
PAH contribution to FUV heating.

Besides the PAH abundance, photoelectric heating rates depend on the local size distribution and amount of grains, which can be spatially variable in PPDs owing to the effects of dust growth, settling, and entrainment (Owen et al. 2011; Hutchison et al. 2016b.a ). Actually, their variabilities are detected in several PPDs (e.g., Pinte et al. 2016). Disk opacity is varied according to the spatial distribution of dust grains, which also strongly affect photoevaporation rates. Hence, in order to derive FUV photoevaporation rates accurately, it is necessary to take account of different spatial distributions of grains with various sizes as well as the reduced abundances of smaller grains.

FUV and X-ray luminosities of young stars are also uncertain factors. A large fraction of FUV photons are considered to be produced within accretion shocks around a classical T Tauri star (CTTS). CTTSs have a wide variety of FUV luminosities with $10^{-6} L_{\odot} \lesssim L_{\mathrm{FUV}} \lesssim L_{\odot}$ 


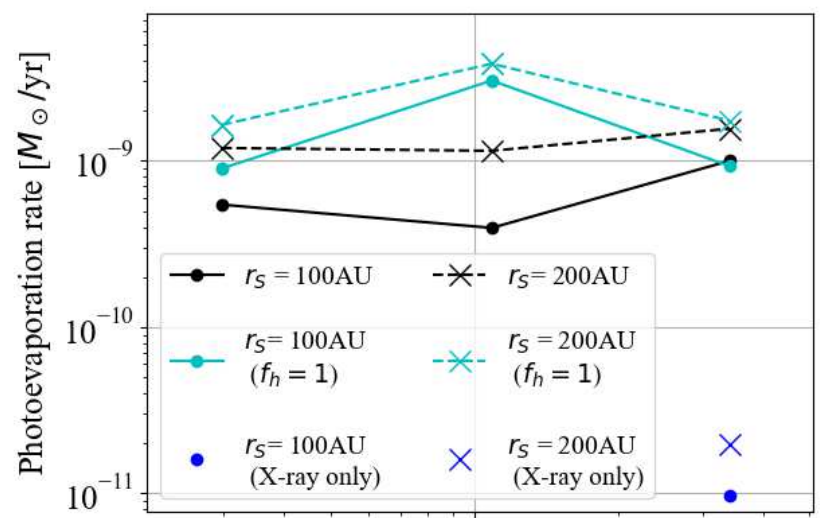

0.1

Cross-section-weighted average photon energy [keV]

FIG. 8.- Resulting photoevaporation rates due to EUV and Xray as functions of the cross-section-weighted average photon energy of the soft, intermediate, and fiducial spectra. The black and cyan lines show the photoevaporation rates derived from the simulations where $f_{h}$ is calculated by Eq. A15 and $f_{h}=1$, respectively. The blue points show the photoevaporation rates of Run $\mathrm{X} / \mathrm{Z0}$ (TABLE 2).

which roughly correlates with the accretion rate $\dot{M}_{\text {acc }}$ as $L_{\text {FUV }} \propto \dot{M}_{\text {acc }}$ Gullbring et al. 1998; Yang et al. 2012). X-ray luminosities of T Tauri stars range between $10^{28} \mathrm{erg} \mathrm{s}^{-1} \lesssim L_{\mathrm{X}} \lesssim 10^{31} \mathrm{erg} \mathrm{s}^{-1}$ Güdel et al. 2007; Vidotto et al. 2014), and measured plasma temperatures are typically $(5-30) \times 10^{6} \mathrm{~K}$ which corresponds to the peak X-ray energy of $\sim 1 \mathrm{keV}$ (Güdel \& Nazé 2009; Alexander et al. 2014). A small number of T Tauri stars are known to have "soft X-ray excess" with temperatures of a few million kelvin $(0.3-0.4 \mathrm{keV}$ ) (Güdel \& Nazé 2009). TW Hya, whose X-ray spectrum is adopted in our fiducial model, is one of them. Thus, our fiducial X-ray spectra might be relatively softer than a typical X-ray spectrum of a T Tauri star without the excessive soft Xray. Our results show that X-ray is ineffective to drive photoevaporation in our fiducial model even though the fiducial spectrum contains the soft X-ray excess. Since softer components of a X-ray spectrum play a major role in driving photoevaporative flows (Section 4.1 and Section 4.2), using a typical X-ray spectrum without the soft $\mathrm{X}$-ray excess would not change the conclusion that Xrays are ineffective to drive photoevaporation. We note that X-ray spectra constructed from emission measure data can be different from 'processed' spectra that actually reach PPD surfaces, considering the possibility of screening close to the X-ray source. We also note that the observed FUV/X-ray luminosities and spectra have wide varieties as above; FUV and X-ray luminosities independently vary with evolutional stages of PPDs. Hence, it is worth investigating relative importance of FUV/X-ray in photoevaporation with a variety of luminosities and spectra.

\section{SUMMARY}

We have performed a suite of radiation hydrodynamics simulations of photoevaporating protoplanetary disks to study the metallicity dependence of photoevaporation due to FUV, EUV, and X-ray radiation. Direct comparison between a variety of cases have shown that X-rays alone do not heat disk gas up to sufficiently high temperature to cause a significant photoevaporative mass loss.

Although the net heating effect is unimportant, X-rays effectively ionize the neutral region in a disk. Then the electron abundance in the neutral region is raised, and charged dust grains recombine more efficiently. The FUV photoelectric heating efficiency is increased by the fast recombination, and the temperature in the neutral region becomes higher because of the strengthened FUV heating. Consequently, including the $\mathrm{X}$-ray radiation results in a larger photoevaporation rate, compared with the cases with FUV heating only.

With FUV, EUV, and X-ray radiation, the disk photoevaporation rate increases as metallicity decreases in the range of $Z \gtrsim 10^{-1.5} Z_{\odot}$ because of the reduced opacity of a disk for FUV photons. At $Z \lesssim 10^{-1.5} Z_{\odot}$, dust-gas collisional cooling becomes efficient compared to FUV photoelectric heating, and suppress photoevaporation. In this metallicity range, the strengthening effect of X-rays is crucial to driving FUV photoevaporation. Without X-rays, the FUV heating does not excite photoevaporation and only EUV-driven flows contribute to the mass loss. Therefore, the photoevaporation rate is significantly large in the simulations with very low metallicities if the $\mathrm{X}$-ray effects are incorporated.

We derived the metallicity dependence of the resulting photoevaporation rates. The metallicity dependence of photoevaporation rates due to FUV or strengthened FUV heating is consistent with the observational metallicity dependence of the disk lifetimes. Our model predicts that protoplanetary disks in an extremely low metallicity environment have longer lifetimes than in solar or sub-solar metallicity environments.

We thank Neal Turner, Mario Flock, Uma Gorti, Shuichiro Inutsuka, Kengo Tomida, and Kei Tanaka for fruitful discussions and helpful comments on the paper. We also thank the anonymous referee for giving insightful comments to improve the manuscript. RN has been supported by the Grant-in-aid for the Japan Society for the Promotion of Science (16J03534) and by Advanced Leading Graduate Course for Photon Science (ALPS) of the University of Tokyo. TH appreciates the financial supports by the Grants-in-Aid for Basic Research by the Ministry of Education, Science and Culture of Japan (16H05996). HN appreciates the financial supports by Grants-in-Aid for Scientific Research (25400229). RK acknowledges financial support via the Emmy Noether Research Group on Accretion Flows and Feedback in Realistic Models of Massive Star Formation funded by the German Research Foundation (DFG) under grant no. KU 2849/3-1. All the numerical computations were carried out on Cray XC30 and Cray XC50 at Center for Computational Astrophysics, National Astronomical Observatory of Japan. 


\section{REFERENCES}

Alexander, R., Pascucci, I., Andrews, S., Armitage, P., \& Cieza, L. 2014, Protostars and Planets VI, 475

Alexander, R. D., Clarke, C. J., \& Pringle, J. E. 2004, MNRAS, 354, 71

Bakes, E. L. O., \& Tielens, A. G. G. M. 1994, ApJ, 427, 822

Clarke, C. J., Gendrin, A., \& Sotomayor, M. 2001, MNRAS, 328, 485

Draine, B. T. 2011, Physics of the Interstellar and Intergalactic Medium

Ercolano, B., \& Clarke, C. J. 2010, MNRAS, 402, 2735

Ercolano, B., Clarke, C. J., \& Drake, J. J. 2009, ApJ, 699, 1639

Ercolano, B., Drake, J. J., Raymond, J. C., \& Clarke, C. C. 2008 , ApJ, 688, 398

Fedele, D., van den Ancker, M. E., Henning, T., Jayawardhana, R., \& Oliveira, J. M. 2010, A\&A, 510, A72

Font, A. S., McCarthy, I. G., Johnstone, D., \& Ballantyne, D. R. 2004, ApJ, 607, 890

Geers, V. C., van Dishoeck, E. F., Visser, R., Pontoppidan, K. M., Augereau, J.-C., Habart, E., \& Lagrange, A. M. 2007, A\&A, 476, 279

Gorti, U., Dullemond, C. P., \& Hollenbach, D. 2009, ApJ, 705, 1237

Gorti, U., \& Hollenbach, D. 2004, ApJ, 613, 424

-. 2008, ApJ, 683, 287

-. 2009, ApJ, 690, 1539

Gorti, U., Hollenbach, D., \& Dullemond, C. P. 2015, ApJ, 804, 29

Güdel, M., \& Nazé, Y. 2009, A\&A Rev., 17, 309

Güdel, M., et al. 2007, A\&A, 468, 353

Gullbring, E., Hartmann, L., Briceño, C., \& Calvet, N. 1998, ApJ, 492, 323

Haisch, Jr., K. E., Lada, E. A., \& Lada, C. J. 2001, ApJ, 553, L153

Hernández, J., et al. 2007, ApJ, 662, 1067

Hollenbach, D. 2017, Mem. Soc. Astron. Italiana, 88, 685

Hollenbach, D., Johnstone, D., Lizano, S., \& Shu, F. 1994, ApJ, 428,654

Hutchison, M. A., Laibe, G., \& Maddison, S. T. 2016a, MNRAS, 463,2725

Hutchison, M. A., Price, D. J., Laibe, G., \& Maddison, S. T. 2016b, MNRAS, 461, 742

Kuiper, R., Klahr, H., Dullemond, C., Kley, W., \& Henning, T. 2010, A\&A, 511, A81

Liedahl, D. A., Osterheld, A. L., \& Goldstein, W. H. 1995, ApJ, 438, L115

Liffman, K. 2003, PASA, 20, 337

Maloney, P. R., Hollenbach, D. J., \& Tielens, A. G. G. M. 1996, ApJ, 466, 561

Mamajek, E. E. 2009, in American Institute of Physics Conference Series, Vol. 1158, American Institute of Physics Conference Series, ed. T. Usuda, M. Tamura, \& M. Ishii, 3-10
McElroy, D., Walsh, C., Markwick, A. J., Cordiner, M. A., Smith, K., \& Millar, T. J. 2013, A\&A, 550, A36

Mewe, R., Gronenschild, E. H. B. M., \& van den Oord, G. H. J. 1985, A\&AS, 62, 197

Meyer, M. R., Backman, D. E., Weinberger, A. J., \& Wyatt, M. C. 2007, Protostars and Planets V, 573

Nakatani, R., Hosokawa, T., Yoshida, N., Nomura, H., \& Kuiper, R. 2018, ApJ, 857, 57

Nomura, H., Aikawa, Y., Tsujimoto, M., Nakagawa, Y., \& Millar, T. J. 2007, ApJ, 661, 334

Oliveira, I., et al. 2010, ApJ, 714, 778

Omukai, K. 2000, ApJ, 534, 809

Owen, J. E., Clarke, C. J., \& Ercolano, B. 2012, MNRAS, 422, 1880

Owen, J. E., Ercolano, B., \& Clarke, C. J. 2011, MNRAS, 411, 1104

Owen, J. E., Ercolano, B., Clarke, C. J., \& Alexander, R. D. 2010, MNRAS, 401, 1415

Pinte, C., Dent, W. R. F., Ménard, F., Hales, A., Hill, T., Cortes, P., \& de Gregorio-Monsalvo, I. 2016, ApJ, 816, 25

Pollack, J. B., Hollenbach, D., Beckwith, S., Simonelli, D. P., Roush, T., \& Fong, W. 1994, ApJ, 421, 615

Ribas, Á., Merín, B., Bouy, H., \& Maud, L. T. 2014, A\&A, 561, A 54

Shull, J. M., \& van Steenberg, M. E. 1985, ApJ, 298, 268

Takasao, S., Tomida, K., Iwasaki, K., \& Suzuki, T. K. 2018, ApJ, 857,4

Tanaka, K. E. I., Nakamoto, T., \& Omukai, K. 2013, ApJ, 773, 155

Vicente, S., Berné, O., Tielens, A. G. G. M., Huélamo, N., Pantin, E., Kamp, I., \& Carmona, A. 2013, ApJ, 765, L38

Vidotto, A. A., et al. 2014, MNRAS, 441, 2361

Wang, L., \& Goodman, J. 2017, ApJ, 847, 11

Wilms, J., Allen, A., \& McCray, R. 2000, ApJ, 542, 914

Yan, M., Sadeghpour, H. R., \& Dalgarno, A. 1998, ApJ, 496, 1044 -. 2001, ApJ, 559, 1194

Yang, H., et al. 2012, ApJ, 744, 121

Yasui, C., Kobayashi, N., Saito, M., \& Izumi, N. 2016a, AJ, 151, 115

Yasui, C., Kobayashi, N., Tokunaga, A. T., Saito, M., \& Izumi, N. 2016b, AJ, 151, 50

Yasui, C., Kobayashi, N., Tokunaga, A. T., Saito, M., \& Tokoku, C. 2009, ApJ, 705, 54

—. 2010, ApJ, 723, L113

\section{APPENDIX}

\section{A. IMPLEMENTATION OF X-RAY HEATING/IONIZATION}

\section{A.1. Cross-Section}

In general, X-rays are absorbed by both non-metal and metal elements which compose both gas and dust grains in a medium. The cross section of the dust grains for X-rays typically contributes relatively small to the total cross section of the medium (Wilms et al. 2000). Hence, we ignore the contribution of the dust grains to the cross section for X-rays in this study.

We use the total cross section presented by Gorti \& Hollenbach (2004) (hereafter, GH04). GH04 calculates the total cross section for a solar metallicity disk and gets a fitted total cross section per hydrogen nuclei

$$
\sigma_{\mathrm{GH} 04}(E)=1.2 \times 10^{-22}\left(\frac{E}{1 \mathrm{keV}}\right)^{-2.594} \mathrm{~cm}^{2},
$$

where $E$ is the X-ray photon energy. As metallicity increases (decreases), the contribution of the metal elements to Eq. (A1) increases (decreases) in the energy range of $E \geq 0.29 \mathrm{keV}$, where $0.29 \mathrm{keV}$ corresponds to the threshold energy 
for carbon ionization (Wilms et al. 2000). Therefore, we modify Eq. (A1) to a metallicity-dependent cross section

$$
\begin{aligned}
& \sigma_{\mathrm{H}}=11.55 \times 10^{-24}\left(\frac{E}{1 \mathrm{keV}}\right)^{-3.4} \mathrm{~cm}^{2}, \\
& \sigma=\left\{\begin{array}{ll}
\sigma_{\mathrm{H}} & (0.1 \mathrm{keV} \leq E \leq 0.29 \mathrm{keV}) \\
\max \left(\sigma_{\mathrm{H}}, \sigma_{\mathrm{GH} 04} \times \frac{Z}{Z_{\odot}}\right) \mathrm{cm}^{2} \quad(E \geq 0.29 \mathrm{keV})
\end{array},\right.
\end{aligned}
$$

where $\sigma_{\mathrm{H}}$ is the hydrogen cross section per hydrogen nuclei presented by GH04.

The ionization cross section of helium is about four times larger than that of hydrogen (Shull \& van Steenberg 1985; Gorti \& Hollenbach 2004), and thus helium contributes to X-ray absorption. However, since helium abundance is typically ten times smaller than hydrogen, including helium contribution to Eq. A3 raises the absorption rate by only $\lesssim 40 \%$. Therefore, in our chemistry model, we neglect the helium contribution to X-ray absorption in order to save computational cost for calculating the chemistry and use Eq. (A3) as the cross section for X-rays.

\section{A.2. Spectral Energy Distribution of X-rays}

For the spectral energy distribution (SED) of X-rays, we use the SED presented in Nomura et al. (2007). The SED is that of TW Hya which is one of the classical T Tauri stars. We set the minimum energy of the X-rays to $E_{\min }=0.1 \mathrm{keV}$ and the maximum energy of it to $E_{\max }=10 \mathrm{keV}$. The absolute values of the SED are normalized so that the total X-ray luminosity is calculated to be $L_{\mathrm{X}}=1 \times 10^{30} \mathrm{erg} \mathrm{s}^{-1}$.

\section{A.3. X-ray Ionization}

When X-rays are absorbed by elements, photoelectrons are ejected from the absorbers. The photoelectrons are called primary electrons. The energy of the primary electrons is so high that they further ionize the ambient neutral gas. This is so-called secondary ionization and the ejected electrons by secondary ionization are called secondary electrons.

In this study, we implement the X-ray ionization of atomic and molecular hydrogen, who are the most abundant species. The total number of the primary electrons per unit volume is

$$
N_{\text {prim }}=\int_{E_{\min }}^{E_{\max }} d E \sigma n_{\mathrm{H}} \frac{F(E)}{E} e^{-\tau_{\mathrm{x}}},
$$

where $\tau_{\mathrm{X}}$ is the optical depth for X-rays defined as $\tau_{\mathrm{X}}=\sigma N_{\mathrm{H}}$. For atomic hydrogen, the amount of energy which goes to secondary ionization is $\Phi_{\mathrm{H}}\left(E-E_{\mathrm{th}}\right)$, where $\Phi_{\mathrm{H}}$ is the fraction of primary electron energy consumed by secondary ionization and $E_{\mathrm{th}}$ is the threshold energy of the ejector of a primary electron. The threshold energy is assumed to typically be much smaller than the ejected non-thermal electron energy. In that case, the number of secondary ionizations can be calculated as $\Phi_{\mathrm{H}} E / 13.6 \mathrm{eV}$. Consequently, the total number of secondary ionizations per unit volume is

$$
\xi_{\mathrm{H}, \mathrm{sec}}=\int_{E_{\min }}^{E_{\max }} d E \frac{F(E)}{E} e^{-\tau_{\mathrm{X}}} \sigma n_{\mathrm{H}}\left(\frac{\Phi_{\mathrm{H}} E}{13.6 \mathrm{eV}}\right),
$$

while the total number of photoionization per unit volume is

$$
\xi_{\mathrm{H}, \operatorname{prim}}=\int_{E_{\min }}^{E_{\max }} d E \frac{F(E)}{E} e^{-\tau_{\mathrm{X}}} \sigma_{\mathrm{H}} n_{\mathrm{HI}} .
$$

Hence, the total ionization rate of atomic hydrogen by X-rays is

$$
\begin{aligned}
R_{\mathrm{X}, \mathrm{H}} & =\left(\xi_{\mathrm{H}, \operatorname{prim}}+\xi_{\mathrm{H}, \mathrm{sec}}\right) / n_{\mathrm{H}} \\
& =\int_{E_{\min }}^{E_{\max }} d E \frac{F(E)}{E} e^{-\tau_{\mathrm{X}}}\left[\sigma_{\mathrm{H}} y_{\mathrm{HI}}+\sigma\left(\frac{\Phi_{\mathrm{H}} E}{13.6 \mathrm{eV}}\right)\right] .
\end{aligned}
$$

In general, the energy fraction consumed by secondary ionization $\Phi_{\mathrm{H}}$ depends on electron abundance and primary electron energy (Maloney et al. 1996), but it is usually simplified as the function which depends only on electron abundance. When electron abundance is sufficiently low such as $y_{\mathrm{e}} \lesssim 0.01$, approximately $35 \%$ of the primary electron energy goes into secondary ionization and thus $\Phi_{\mathrm{H}}$ can be approximated by $\Phi_{\mathrm{H}} \simeq 0.35$ (Maloney et al. 1996; Gorti \& Hollenbach 2004). In a protoplanetary disk, even if X-rays ionize the medium, electron abundance is typically low $y_{\mathrm{e}} \ll 1$ except the H II region where EUV photons ionize the gas. In the H II region, the electron abundance is $y_{\mathrm{e}} \sim 1$ and the atomic hydrogens are almost completely ionized $\left(y_{\mathrm{HI}} \sim 0\right)$. The approximation $\Phi_{\mathrm{H}} \simeq 0.35$ is not appropriate in this case. As electron abundance increases, a smaller amount of energy goes into secondary ionization. In order to simply incorporate this effect, we use

$$
\Phi_{\mathrm{H}}=0.35 y_{\mathrm{HI}},
$$


as a first approximation. Hence, Eq. (A7) reduces to

$$
R_{\mathrm{X}, \mathrm{H}}=y_{\mathrm{HI}} \int_{E_{\min }}^{E_{\max }} d E \frac{F(E)}{E} e^{-\tau_{\mathrm{X}}}\left[\sigma_{\mathrm{H}}+\sigma\left(\frac{25.7 E}{1 \mathrm{keV}}\right)\right] .
$$

For the X-ray ionization of molecular hydrogen, we use $2 \sigma_{\mathrm{H}}$ as the cross section of molecular hydrogen as GH04 (cf. Yan et al. 1998; Draine 2011). Note that the ratio of the cross section of a hydrogen molecule to that of a hydrogen atom is $\sim 2.8$ in the high energy limit (Wilms et al. 2000; Yan et al. 2001; Draine 2011). The number of primary ionization for molecular hydrogens is

$$
\xi_{\mathrm{H}_{2}, \operatorname{prim}}=\int_{E_{\min }}^{E_{\max }} d E \frac{F(E)}{E} e^{-\tau_{\mathrm{X}}}\left(2 \sigma_{\mathrm{H}}\right) n_{\mathrm{H}_{2}} .
$$

Regarding the secondary ionization, the fraction of primary electron energy going into secondary ionization is similar to that of atomic hydrogens if electron abundance is small (Maloney et al. 1996). Thus, $\Phi_{\mathrm{H}}$ is used also for secondary ionization of molecular hydrogens. The number of the secondary ionization of $\mathrm{H}_{2}$ per photoelectron is changed from Eq. (A5) by the difference between the ionization energies of an atomic and molecular hydrogen. We calculate the secondary ionization of molecular hydrogens as

$$
\begin{aligned}
\xi_{\mathrm{H}_{2}, \mathrm{sec}}=2 y_{\mathrm{H}_{2}} \int_{E_{\min }}^{E_{\max }} & d E \frac{F(E)}{E} e^{-\tau_{\mathrm{X}}} \sigma n_{\mathrm{H}} \\
& \times\left(\frac{13.6 \mathrm{eV}}{15.4 \mathrm{eV}}\right)\left(\frac{25.7 E}{1 \mathrm{keV}}\right) .
\end{aligned}
$$

The first factor $2 y_{\mathrm{H}_{2}}$ is used instead of $y_{\mathrm{HI}}$ in Eq. (A8). Since the maximum value of $y_{\mathrm{H}_{2}}$ is 0.5 , the factor becomes unity in Eq. A11 in fully molecular gas. After all, the total ionization rate of molecular hydrogens reduces to

$$
\begin{aligned}
R_{\mathrm{X}, \mathrm{H}_{2}} & =\left(\xi_{\mathrm{H}_{2}}, \operatorname{prim}+\xi_{\mathrm{H}_{2}, \mathrm{sec}}\right) / n_{\mathrm{H}} \\
& =2 y_{\mathrm{H}_{2}} \int_{E_{\min }}^{E_{\max }} d E \frac{F(E)}{E} e^{-\tau_{\mathrm{X}}}\left[\sigma_{\mathrm{H}}+\sigma\left(\frac{22.7 E}{1 \mathrm{keV}}\right)\right] .
\end{aligned}
$$

X-rays also ionize helium by primary and secondary ionization. The fraction of primary electron energy consumed by secondary ionization of helium is $\Phi_{\mathrm{He}} \sim 0.05$ (Shull \& van Steenberg 1985; Gorti \& Hollenbach 2004), which is much less than that of hydrogen. Taking into account this weaker secondary ionization and the fact that helium abundance is an order of magnitude less than hydrogen abundance, helium ionization rates would be smaller than those of hydrogen. Since helium recombination rates and hydrogen recombination rates have only a small difference (about a factor of two in the range of $10 \mathrm{~K} \leq T \leq 1000 \mathrm{~K}$, McElroy et al. 2013), the ionization degree of helium due to X-rays would be smaller than or at least the same order of magnitude as hydrogen ionization degree due to X-rays. Thus, including $\mathrm{X}$-ray ionization of helium in our chemistry might raise electron abundance in the neutral region of a disk by several tens of percent. In the sense that FUV heating is strengthened more by the higher electron abundance as discussed in Section 3.1. Section 3.2, and Section 3.3, taking account of helium ionization due to X-ray would make our conclusion firmer. Nevertheless, it is expected that including helium ionization does not significantly affect our results, and so we do not incorporate helium ionization in our chemistry to save the computational cost for the calculation of the chemistry network.

\section{A.4. X-ray Heating}

The total energy deposited to primary electrons by X-ray absorption is given by

$$
\epsilon_{\mathrm{tot}}=\int_{E_{\min }}^{E_{\max }} d E F(E) e^{-\tau_{\mathrm{X}}} \sigma n_{\mathrm{H}} .
$$

Not all the energy goes into heating due to other processes such as secondary ionization and excitations of the ambient gas. If hydrogens are purely atomic in gas, about $10 \%$ of the deposited energy by the absorption of X-rays results in gas heating (Maloney et al. 1996). If hydrogens are purely molecular, about $40 \%$ of the deposited energy thermalizes. Therefore, in this study, we set the X-ray heating rate to

$$
\begin{aligned}
\Gamma_{\mathrm{X}} & =f_{h} \epsilon_{\mathrm{tot}}, \\
f_{h} & \equiv \frac{0.1 y_{\mathrm{HI}}+0.4 y_{\mathrm{H}_{2}}}{y_{\mathrm{HI}}+y_{\mathrm{H}_{2}}},
\end{aligned}
$$

where $f_{h}$ is the heating efficiency of the X-ray heating rate.

\section{B. CHEMICAL REACTIONS}

We use the same chemistry network as that of Paper I other than we add chemical reactions involving $\mathrm{H}_{2}{ }^{+}$to the network. The added chemical reactions are listed in TABLE B. 
Table B1. Chemical reactions incorporated additionally in our simulations

\begin{tabular}{lllc}
\hline Label & Reaction & Rate Coefficient & Reference \\
\hline \hline $\mathrm{k} 9$ & $\mathrm{H}+\mathrm{H}^{+} \longrightarrow \mathrm{H}_{2}{ }^{+}$ & $10^{-19.38-1.523 \log T+1.118(\log T)^{2}-0.1269(\log T)^{3}}$ & 1 \\
k10 & $\mathrm{H}_{2}++\mathrm{H} \longrightarrow \mathrm{H}_{2}+\mathrm{H}^{+}$ & $6.4 \times 10^{-10}$ & 1 \\
k11 & $\mathrm{H}_{2}+\mathrm{H}^{+} \longrightarrow \mathrm{H}+\mathrm{H}_{2}{ }^{+}$ & $3.0 \times 10^{-10} \exp \left[-2.1050 \times 10^{4} \mathrm{~K} / T\right]$ & 1 \\
p4 & $\mathrm{H}+\gamma \longrightarrow \mathrm{H}^{+}+\mathrm{e}$ & $R_{\mathrm{X}, \mathrm{H}} \quad$ (cf. Eq. E9) & 2 \\
p5 & $\mathrm{H}_{2}+\gamma \longrightarrow \mathrm{H}_{2}+\mathrm{e}$ & $R_{\mathrm{X}, \mathrm{H}_{2}} \quad$ (cf. Eq. (A12) & 2 \\
\hline
\end{tabular}

Reference — (1) Omukai (2000) (2) Gorti \& Hollenbach (2004) 